\title{
Truncating and Oversampling OFDM Signals in White Gaussian Noise Channels
}

\author{
Waseem Ozan ${ }^{+}$, Kyle Jamieson ${ }^{\# *}$ and Izzat Darwazeh ${ }^{+}$ \\ ${ }^{+}$Department of Electronic and Electrical Engineering and ${ }^{\#}$ Computer Science, University College London, London, UK \\ ${ }^{*}$ Department of Computer Science, Princeton University, Princeton, NJ 08540, USA \\ Email: \{waseem.ozan.13, k.jamieson, i.darwazeh\}@ucl.ac.uk
}

\begin{abstract}
This work introduces a modified version of the orthogonal frequency division multiplexing (OFDM) signal by truncating OFDM symbols in the time domain. Sub-carriers are no longer orthogonally packed in the frequency domain as time samples are only partially transmitted, leading to improved spectral efficiency. In this work, mathematical expressions are derived for the newly proposed Truncated OFDM (TOFDM) signal, followed by interference analysis and performance comparisons. We also consider optimal and practical decoder architectures. Results from a Sphere Decoder-based decoder indicate that truncation length can significantly affect the error performance. With short truncation length, using a purpose designed detector, signals can be recovered even with truncated symbol transmission.
\end{abstract}

Index Terms-Multicarrier modulation; Truncated OFDM; SEFDM.

\section{INTRODUCTION}

OFDM systems are a subset of multi carrier modulation (MCM) systems, where the different subcarriers in OFDM maintain orthogonality and where the data rate on each subcarrier is a fraction of the overall data rate. Orthogonality in OFDM is a crucial factor in its successful operation: loss of orthogonality leads to interference and deterioration in bit error rate [1]. Orthogonality, in time and frequency, is maintained by ensuring that the spacing between the subcarriers equals the inverse of the symbol rate.

Spectrum scarcity and the need to send data at higher bit rates has led to an increase in research into systems that use spectrum more efficiently. Recently a set of timeand frequency-domain techniques has been proposed to enhance spectrum efficiency by deliberate violation of the orthogonality criterion in multicarrier systems. During the last decade, spectrally-efficient frequency division multiplexing (SEFDM) signals and systems have been proposed as a means to economize on bandwidth, yet avoid intolerable degradation of bit error rates, relative to OFDM [2], [3], [4]. Such is achieved in SEFDM by decreasing the distance between subcarriers in the frequency domain while keeping the same data rate on each subcarrier [3], [4]. Similarly, multi-stream faster-than-Nyquist (FTN) systems have been suggested to improve spectral efficiency by transmitting at a higher rate data than imposed by the Nyquist limit for the same bandwidth [5][7][8]. FTN fulfils this by signaling on each subcarrier at a rate higher than the frequency separation between two adjacent subcarriers, effectively overlapping symbols in time at the expense of loss of orthogonality [5]. It is worth noting that systems that overlap multicarrier symbols in time, without loss of orthogonality, were reported in the 1990s such as the time-limited system of Li and Slette reported in [6]. Another reported non-orthogonal multicarrier system is termed high compaction multi carrier modulation (HC-MCM), based on a similar concept to SEFDM and FTN, where enhanced spectral efficiency is achieved by either reducing the subcarrier spacing or decreasing the transmission time for OFDM symbols [9].

The work reported here proposes a different non-orthogonal system format which modifies OFDM signals by truncating (as opposed to overlapping) the end of each OFDM symbol, in a way similar to what was studied in the context of assessing the spectrum of oversampled OFDM and SEFDM signals [4]. As such, this new format is termed truncated OFDM (TOFDM). Here, each OFDM time domain symbol is truncated by a factor $(\gamma)$ to generate the TOFDM signal. Therefore, time samples are only partially transmitted and part of the symbol is lost at transmission. Furthermore, the fact that the TOFDM symbol time is shorter than that of an OFDM one, implies loss of orthogonality and consequently the introduction of inter-carrier interference (ICI) amongst the subcarriers. In SEFDM, the issue of ICI was dealt with through the application of different techniques, such as orthonormalization [2], optimum detection using maximum likelihood (ML) detectors [2][3][10], Sphere decoders [3][11] and iterative interference cancellation [12][13]. To illustrate the utility of TOFDM signals, a sphere decoder (SD) is used in this work as it offers performance close to that of ML with much reduced complexity and computation time.

The work in this paper focuses on studying the parameters that affect the error performance and complexity in a proposed TOFDM system. Therefore, mathematical derivations for TOFDM signals and the characterization of its ICI are presented and system performance is studied through computer simulations. Results show that the TOFDM ICI levels depend on two key signal parameters: the truncation factor and the oversampling factor.

In this paper, Section II depicts the TOFDM signal model. 


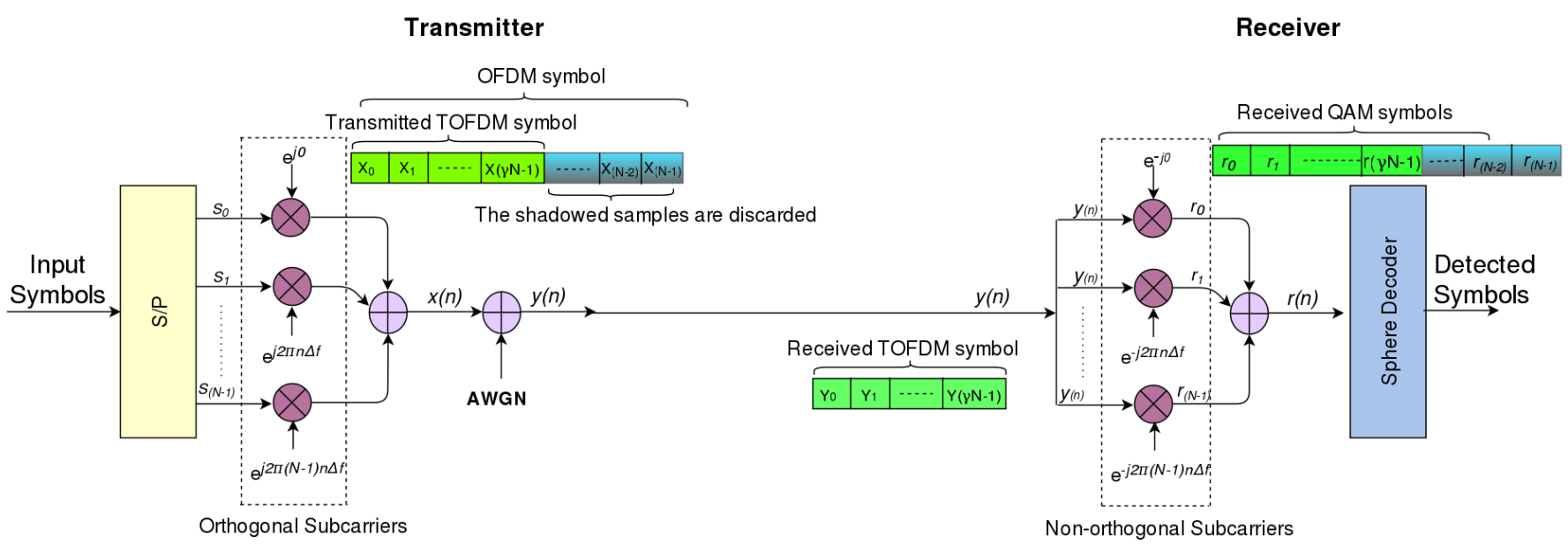

Fig. 1. TOFDEM simplified system modem.

Section III derives the characterization of ICI in TOFDM signals. Section IV provides the description of an adopted detection method TOFDM, and Section V concludes the paper.

\section{TRuncated OFDM Signal Model}

This section first describes the basic signal model for TOFDM signals, secondly depicts the methodology of implementing the TOFDM system and then compares TOFDM with other similar spectrally efficient techniques.

\section{A. Signal Model}

A TOFDM symbol $x(t)$ is generated by multiplexing a vector of $N$ inputs of a complex signal denoted as $S$ onto parallel subcarriers, in a similar way to OFDM but with a time truncation factor, $\gamma$, as :

$$
x(t)=\frac{1}{\sqrt{T_{T O F D M}}} \sum_{l=-\infty}^{\infty} \sum_{n=0}^{N-1} S_{l, n} \exp \left(\frac{j 2 \pi\left(t-l \gamma T_{O F D M}\right)}{T_{O F D M}}\right),
$$

Defining $T_{T O F D M}$ as the TOFDM symbol duration as $T_{T O F D M}=\gamma T_{O F D M}$.

Therefore, (1) can be represented as:

$$
x(t)=\frac{1}{\sqrt{T_{T O F D M}}} \sum_{l=-\infty}^{\infty} \sum_{n=0}^{N-1} S_{l, n} \exp \left(\frac{j 2 \pi\left(t-l T_{T O F D M}\right)}{T_{O F D M}}\right),
$$

where $S_{l, n}$ denotes the data symbol modulated on the $n^{\text {th }}$ subcarrier and $l^{\text {th }}$ TOFDM symbol, $N$ represents the index of the subcarrier and $1 / \sqrt{T_{O F D M}}$ is a normalization factor. The frequency spacing between two adjacent subcarriers in TOFDM signals is defined as $1 / T_{T O F D M}$, which is equal to that of OFDM signals. However, the duration of each TOFDM symbol, in comparison with OFDM symbols, is shorter by the factor $\gamma$. Therefore, the subsequent symbol comes earlier in time by $(1-\gamma)$ because of the truncation in the preceding symbol. For example, in the case of using digital signal, if $N=10$ and, $\gamma=0.8$, the number of transmitted samples per TOFDM symbol is eight while there are ten samples for an OFDM symbol. Thus, five TOFDM symbols may be sent in the same duration as four OFDM ones, carrying the same data. Nevertheless, the TOFDM symbol duration is not equal to the reciprocal of the frequency spacing between the subcarriers, which means the generated TOFDM signal are not orthogonal, where the transmission rate of each subcarrier, in comparison with OFDM signal, is higher.

Fig. 1 illustrates a basic block diagram for the TOFDM system model used in this study. The figure sketches a simple time domain representation of TOFDM Transmitted and received symbols, showing partial transmission for the samples, as the shadowed samples are not transmitted. Simply, OFDM symbols are generated and then truncated before transmission. At the receiver, the duration of the symbol at each modulator is shorter by the factor $\gamma$, relative to the original OFDM symbol. Note that $\gamma N$ is equal to the minimum number of the transmitted samples per TOFDM symbol.

By sampling one TOFDM symbol with sample period $T_{O F D M} / Q$, where $Q=\rho N$ and $\rho \geq 1$ is the oversampling rate, a discrete TOFDM symbol is expressed as [14]:

$$
X(k)=x\left(\frac{k T_{O F D M}}{Q}\right)=\frac{1}{\sqrt{\gamma Q}} \sum_{n=0}^{Q-1} S_{n} \exp \left(\frac{j 2 \pi n k}{Q}\right),
$$

where $k$ is the index of the time sample of the TOFDM symbol for the range $0 \leq k \leq \gamma Q-1$. Equation (3) depicts the process of modulating the input data onto different subcarriers in a TOFDM symbol, it is written in matrix form as [14], 


$$
X=\boldsymbol{\Phi} * S .
$$

$X=\left[x_{0}, x_{1}, \ldots, x_{\gamma Q-1}\right]^{T}$ represents a $\gamma Q$-dimensional vector that is a sampled TOFDM symbol in the time domain, $S=\left[s_{0}, s_{1}, \ldots, s_{\gamma Q-1}\right]^{T}$ as an $N$-dimensional vector that is a sampled input data in the freuency domain. The symbol $\boldsymbol{\Phi}$ signifies the sampled carrier matrix; $\boldsymbol{\Phi}$ is a $\gamma Q \times Q$ two-dimensional matrix. The elements of the $\Phi$ matrix are $\boldsymbol{\Phi}_{k, n}=\frac{1}{\sqrt{\gamma Q}} \exp (j 2 \pi n k / Q)$, where $0 \leq k \leq Q-1$ and $0 \leq k \leq \gamma Q-1$. The symbol [.] $]^{T}$ refers to the matrix or alternatively the vector transpose.

\section{B. TOFDM Signal Representation vs OFDM, SEFDM and FTN}

As mentioned before, every OFDM symbol is truncated to generate the TOFDM symbol, which means the TOFDM symbol width is shorter than the OFDM symbol by a factor $\gamma$, where only a $\gamma T_{O F D M}$ duration of the signal is sent while the remaining $(1-\gamma) T_{O F D M}$ is discarded. In the digital domain this means only a partial number of samples of OFDM symbol is sent. The TOFDM symbol is represented in terms of the OFDM symbol as follows:

$$
\begin{aligned}
& \text { TOFDM symbol }\left(T_{\text {TOFDM }}\right)= \\
& \qquad w(t) * O F D M \text { symbol }\left(T_{O F D M}\right),
\end{aligned}
$$

where $w(t)$ is a time-domain rectangular window with interval $=\gamma$.

$$
w(t)=\left[\begin{array}{ll}
1 & , 0 \leq t \leq \gamma \\
0 & , \gamma \leq t \leq 1
\end{array}\right],
$$

where $\gamma \leq 1$ When $\gamma=1$, the symbol is an OFDM symbol, whereas lesser values of $\gamma$ achieve different truncations. A lesser value of $\gamma$ offers a greater spectral efficiency in the time domain. This implies that more data can be sent over the same frequency bandwidth. Equation (6) shows there is no change in the power during the interval, which means the function of the window is only truncating in time domain. In Fig. 2, it is clear that OFDM and SEFDM symbols are sent with the same symbol duration, while FTN transmits overlapped symbols. OFDM systems transmit orthogonal symbols in time and frequency, while SEFDM systems transmit more data by relaxing the spacing between the subcarriers in the frequency domain, which means the bandwidth of these systems is narrower than OFDM despite no difference in the time duration of SEFDM and OFDM symbols. FTN systems send more data on the same bandwidth that is used by OFDM system by transmitting symbols faster than the Nyquist limit, which makes FTN symbols shorter in the time domain. This allows more symbols to be sent in a shorter time, equivalently saving bandwidth. However, due to transmission at a higher rate, the FTN signal will overlap and produce interference. On the other hand, TOFDM sends partial samples of the OFDM symbol, although TOFDM has the same symbol duration as FTN, the features of these symbols are totally different as explained earlier. However, truncation in the time domain can lead to

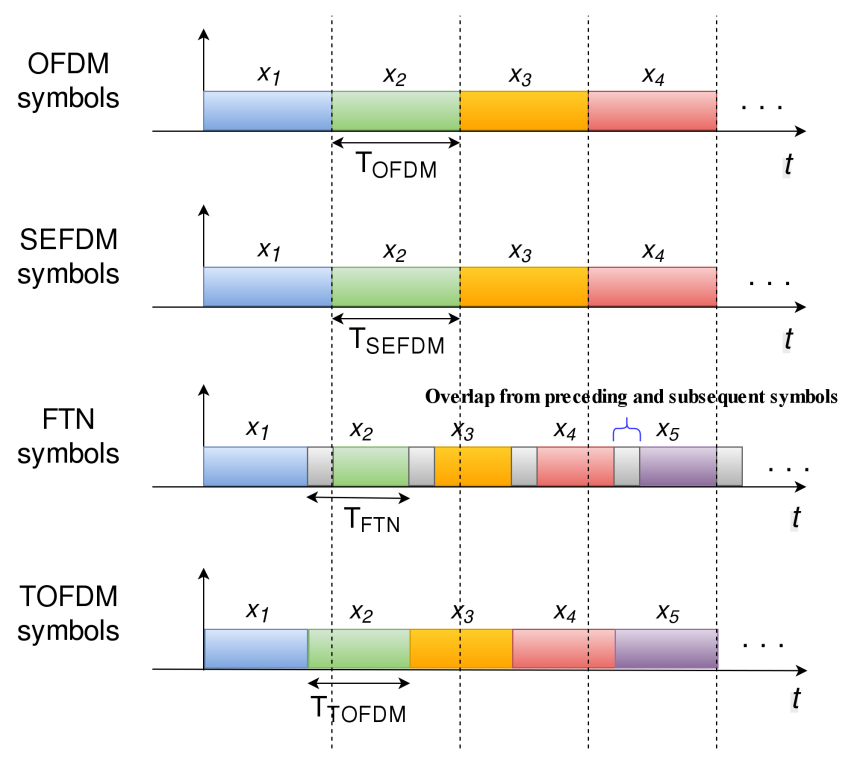

Fig. 2. Conceptual illustrations of OFDM, SEFDM, FTN and TOFDM in time domain where the compression factor for FTN and TOFDM is $\gamma=0.8$.

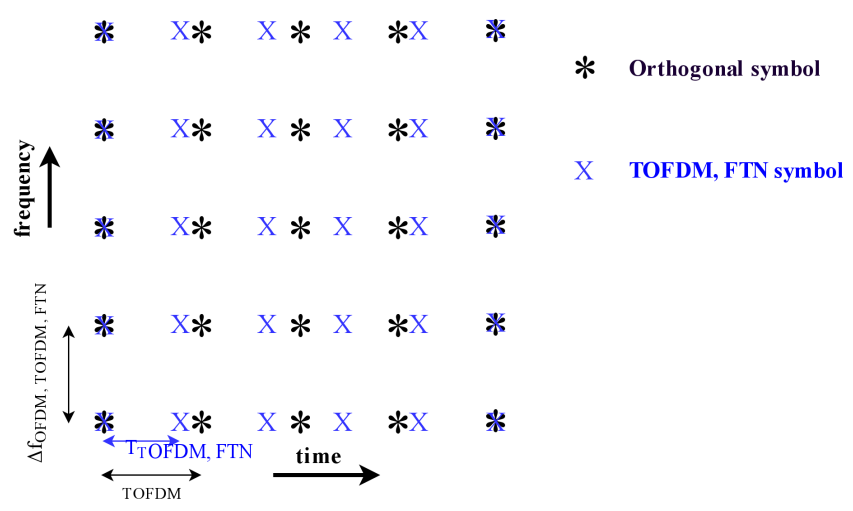

Fig. 3. Conceptual illustration of OFDM, TOFDM and FTN in frequency and time.

lost information, and also generates interference between the subcarriers.

In Fig. 3, the frequency spacing between the subcarriers of OFDM, TOFDM and FTN signals are the same, which means all the subcarriers are positioned at the same frequencies when TOFDM signals are generated. However, the symbol duration of TOFDM and FTN is shorter in time domain, which implies that the transmission rate is higher. In addition, because the symbol becomes shorter, the bandwidth of each subcarrier becomes wider; the signals are changed to be non-orthogonal which leads to interference in the system.

In Fig. 4, OFDM, TOFDM and FTN have the same frequency spacing while SEFDM has a compressed bandwidth. The bandwidth savings in SEFDM has been accomplished by compressing the spacing in the frequency domain between subcarriers. It can be noticed that SEFDM has the same symbol duration (width in the figure) as 


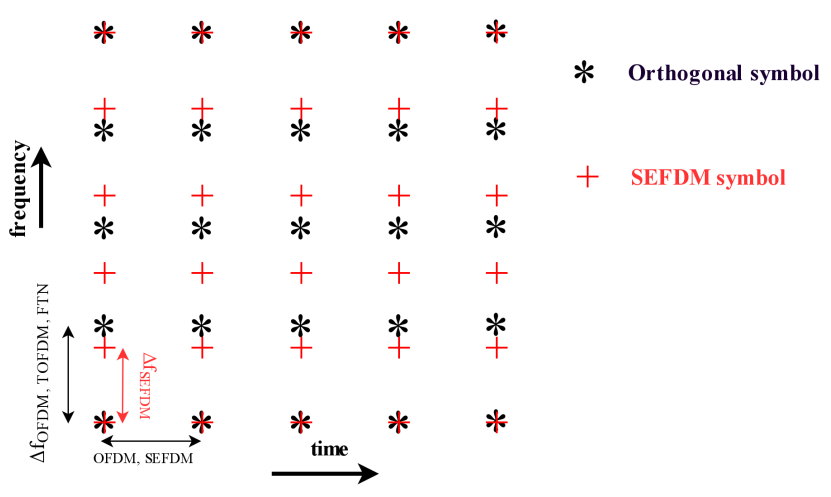

Fig. 4. Conceptual illustration of OFDM and SEFDM in frequency and time.

OFDM. Although the SEFDM system has the same symbol duration as OFDM, the system produces interference due to a compression in the frequency domain, which makes the subcarriers closer.

\section{Characterising The ICI In The TOFDM System}

A similar method of analysis to that developed for SEFDM in [13] is followed in this treatment. Sampling the subcarriers of a TOFDM symbol results in the carrier matrix, denoted as $\boldsymbol{\Phi}$, which represents the mathematical function of a sampled bank of modulators:

$$
\boldsymbol{\Phi}=\frac{1}{\sqrt{\gamma Q}}\left[\begin{array}{ccccc}
1 & 1 & 1 & \ldots & 1 \\
1 & e^{j \frac{2 \pi}{Q}} & e^{j \frac{4 \pi}{Q}} & \cdots & e^{j \frac{2 \pi(Q-1)}{Q}} \\
\vdots & \vdots & \vdots & \ddots & \vdots \\
1 & e^{j \frac{2 \pi(\gamma Q-1)}{Q}} & e^{j \frac{4 \pi(\gamma Q-1)}{Q}} & \cdots & e^{j \frac{2 \pi(\gamma Q-1)(Q-1)}{Q}}
\end{array}\right]_{\gamma Q \times Q}
$$

In order to study and understand the inter carrier interference (ICI) in a TOFDM system, the cross correlation matrix, denoted as $\mathrm{C}$, is now evaluated.

We assume a discrete representation of the signal, as this allows the study of truncation and oversampling's effects on generated signal and detection properties. We follow a similar method to that reported in [14].

Cross correlation is a method used to evaluate to which degree two signals are correlated. Assuming that the two discretized TOFDM sub-carriers are $e^{\frac{2 \pi k m}{Q}}$ and $e^{\frac{2 \pi k n}{Q}}$, the cross correlation $c[m, n]$ of two arbitrary subcarriers $m$ and $n$ in a TOFDM signal may be represented by:

$$
\begin{aligned}
c[m, n] & =\frac{1}{\gamma Q} \sum_{k=0}^{\gamma Q-1} \exp \left(\frac{-j 2 \pi m k}{Q}\right) \exp \left(\frac{j 2 \pi n k}{Q}\right) \\
& =\frac{1}{\gamma Q}\left[\frac{1-e^{-j 2 \pi(m-n) \gamma}}{1-e^{\frac{-j 2 \pi(m-n)}{Q}}}\right],
\end{aligned}
$$

where $k=0, \ldots,(\gamma Q-1)$, and $Q$ is the number of samples per subcarrier, and the $(m-n)$ represents the carriers index distance, that is the index distance between a subcarrier $m$ to subcarrier $n$. Therefore, each correlation coefficients $c[m, n]$ in (8) above is an interference between two subcarriers. Since the condition of orthogonality between all subcarriers is violated, each subcarrier contributes some level of interference to produce the overall ICI in the signal.

The derivation of the third line of (8) is based on the sum of geometric series: $\sum_{k=0}^{Q-1} r^{k}=\frac{1-r^{Q}}{1-r}$. The correlation matrix $\mathbf{C}$, given in (9) below, is the interference matrix, whose elements are those derived in (8) above, giving the spillage of energy (i.e. interference) among all subcarriers as well as the relative value of each subcarrier

$$
C=\frac{1}{\gamma Q}\left[\begin{array}{cccc}
\gamma Q & \frac{1-e^{j 2 \pi \gamma}}{1-e^{\frac{j 2 \pi}{Q}}} & \cdots & \frac{1-e^{j 2 \pi(Q-1) \gamma}}{1-e^{\frac{j 2 \pi(Q-1)}{Q}}} \\
\frac{1-e^{-j 2 \pi \gamma}}{1-e^{\frac{-j 2 \pi}{Q}}} & \cdots & \frac{1-e^{j 2 \pi(Q-2) \gamma}}{1-e^{\frac{j 2 \pi(Q-2)}{Q}}} \\
\vdots & \vdots & \ddots & \vdots \\
\frac{1-e^{-j 2 \pi(Q-1) \gamma}}{1-e^{\frac{-j 2 \pi(Q-1)}{Q}}} & \frac{1-e^{-j 2 \pi(Q-2) \gamma}}{1-e^{\frac{-j 2 \pi(Q-2)}{Q}}} & \cdots & \gamma Q
\end{array}\right]_{Q \times Q}
$$

The correlation matrix $\mathbf{C}$ is a Toeplitz [15] and Hermitian $Q \times Q$ matrix and its elements are given by:

$$
c[m, n]=\frac{1}{\gamma Q}\left\{\begin{array}{cc}
\gamma Q & , m=n \\
\frac{1-e^{-j 2 \pi(m-n) \gamma}}{1-e^{\frac{-j 2 \pi(m-n)}{Q}}} & , m \neq n
\end{array}\right\}
$$

It is evident that the non-diagonal elements in the matrix $\mathbf{C}$ are non-zero indicating the self-created inter carrier interference in TOFDM. The correlation matrix is used to estimate the interference among the subcarriers. Although only partial data is transmitted and the rest is discarded, the receiver has the ability to retrieve the data by estimating the removed part of the symbol by using the correlation matrix. Examination of (8) shows that the correlation matrix can be found by using subcarrier matrix as they are directly related by

$$
\mathbf{C}=\boldsymbol{\Phi}^{*} \times \boldsymbol{\Phi},
$$

where $[.]^{*}$ is the Hermitian transpose operation. Equation (11) shows that the correlation matrix $\mathbf{C}$ can be generated easily by using the carrier matrix $\Phi$, where the latter can be found by knowing the truncation and the oversampling factors.

\section{Signal Detection And Results}

This section starts with describing the detection stage at the receiver side and then shows the BER results. 


\section{A. The Detection Stage}

We follow detection techniques similar to those applied to SEFDM in [3]. The transmitted TOFDM signal, $x(t)$ is contaminated with Additive White Gaussian Noise (AWGN) signal, $w n(t)$ to give the received signal $y(t)$ as:

$$
y(t)=x(t)+w n(t)
$$

At the receiver $y(t)$ is correlated with the conjugate subcarrier to demodulate the received signal. The discrete form of the received signal is expressed as:

$$
R(k)=\frac{1}{\sqrt{\gamma Q}} \sum_{n=0}^{\gamma Q-1} x_{n} \exp \left(\frac{-j 2 \pi n k}{Q}\right)+W^{*}{ }_{c}(k),
$$

where $k=0, \ldots, Q-1, x_{n}$ is the transmitted signal, $R$ is the received signal after demodulation and $W^{*}{ }_{c}$ is a $Q \times 1$ vector of AWGN samples correlated with the conjugate subcarriers. The received signal is then fed to a SD to detect the signal. The mathematical representation for the SD is expressed as:

$$
S_{S D}=\arg \min _{S \in M^{Q},\|R-\mathbf{C} S\|^{2} \leq g}\|R-\mathbf{C} S\|^{2},
$$

where $S_{S D}$ is the estimated signal, $M^{Q}$ is the all possible combinations for the transmitted TOFDM symbols, $M$ is the cardinality of the constellation diagram, $g$ is the radius of the hypersphere search area that is centered at the vector $R$, and $S$ is the vector that has the highest probability of being transmitted. The latter is found by finding the minimum Euclidean distance from all the possible vector combinations which take place inside the multi-dimensional hypersphere searching area. In other words, by using a SD we can detect the received signal by estimating the truncated portion of every TOFDM symbol, enabling recovery of the intended signal. In addition, the SD tests a subset of the all possible combinations of transmitted symbols, which means it enjoys improved computational efficiency over a ML detector, which compares the received symbol with the all possible combinations of the transmitted TOFDM symbols.

\section{B. The BER Analysis}

The TOFDM system is tested using 10 subcarriers, QPSK modulation and with different values of truncation factor $\gamma=$ $0.9,0.8,0.7$ and 0.6 , for different values of oversampling. A smaller value of $\gamma$ allows the system to transmit fewer samples and give a higher spectral efficiency. Two oversampling values ( 1 and 4 ) are used in these simulations, where higher values of oversampling will result in lower levels of aliasing, as described in [15], leading to better bit error rate results.

Figures 5 and 6 show the error performance for different values of, without and with truncation, respectively. The power penalty, defined as $E_{b} / N_{o}$ difference between OFDM

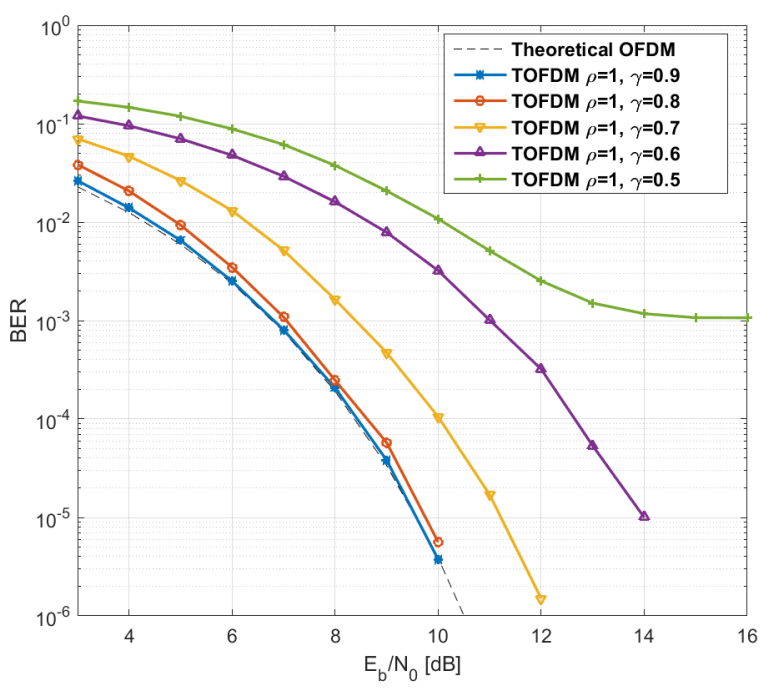

Fig. 5. BER for TOFDM system with no oversampling.

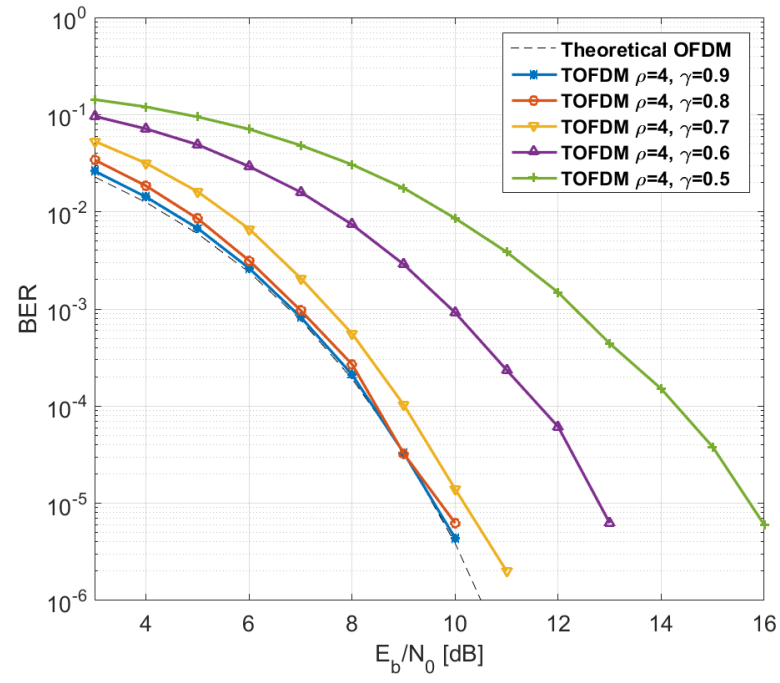

Fig. 6. BER for TOFDM system with oversampling factor $=4$.

and TOFDM systems, is measured at $\mathrm{BER}=10^{-3}$ in Fig. 7. It should be noted that by truncating $20 \%$ of samples, TOFDM shows identical performance as OFDM. With further samples truncation up to $30 \%$, therefore higher interference, $1 \mathrm{~dB}$ power penalty is observed. In terms of improved spectral efficiency, such power penalty may be tolerated. The figure also demonstrates the effect of oversampling on power penalty. For high truncation factors such as 0.9 and 0.8 , the oversampling has no clear effect. For lower truncation factors, therefore higher interference, the power penalty is reduced with the increase of oversampling factors due to improved signal resolutions. 


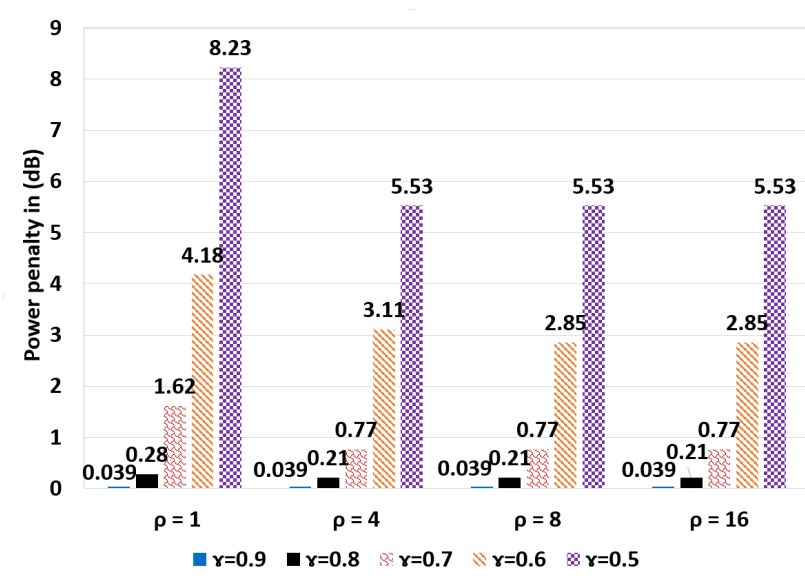

Fig. 7. $E_{b} / N_{o}$ difference between OFDM and TOFDM systems.

\section{CONCLUSION}

In this paper, a modified version of the OFDM signal is proposed by truncating the OFDM symbols in time. The new signal is termed truncated OFDM (TOFDM). A mathematical representation of the signal is presented and TOFDM characteristics are compared to those of SEFDM and FTN signals. Error performance is studied in AWGN channels for different values of truncation and oversampling factors. The reported results show that TOFDM saves bandwidth by sending partial information, yet allows detection with a small deterioration of error rates.

\section{ACKNOWLEDGMENTS}

The research leading to these results has received funding from the European Research Council under the European Unions Seventh Framework Programme (FP/2007-2013) / ERC Grant Agreement no. 279976. We are grateful to UCL for funding Waseem Ozans PhD studies. The authors would like to thank Mr. Tongyang Xu of University College London for his assistance in the Sphere Decoder design and his helpful comments on this work.

\section{REFERENCES}

[1] E. Dahlman, S. Parkvall, and J. Skld, " $4 G$ LTE/LTE-Advanced for mobile broadband”. Oxford : Academic Press, Jan. 2011.

[2] I. Kanaras, A. Chorti, M. R. D. Rodrigues and I. Darwazeh, "Spectrally Efficient FDM Signals: Bandwidth Gain at the Expense of Receiver Complexity,“. Communications, 2009. ICC '09. IEEE International Conference on, Dresden, 2009, pp. 1-6.

[3] S. I. Ahmed, "Spectrally Efficient FDM Communication Signals and Transceivers: Design, Mathematical Modeling and System Optimization, “ Ph.D. thesis, University College London (UCL), 2011.

[4] R. C. Grammenos, "Spectrum Optimisation in Wireless Communication Systems: Technology Evaluation, System Design and Practical Implementation“ Ph.D. thesis, University College London (UCL), 2013.

[5] J. B. Anderson, F. Rusek and V. wall, "Faster-Than-Nyquist Signaling," in Proceedings of the IEEE, vol. 101, no. 8, pp. 1817-1830, Aug. 2013.

[6] R. Li and G. Stette, "Time-limited orthogonal multicarrier modulation schemes, " IEEE Transactions on Communications, 1995, pp. 12691272.

[7] Y. Feng and J. Bajcsy, "Improving Throughput of Faster-than-Nyquist Signaling over Multiple-Access Channels," Vehicular Technology Conference (VTC Spring), 2015 IEEE 81st, Glasgow, 2015, pp. 1-5.
[8] Y. Feng and J. Bajcsy, "On Faster-than-Nyquist Transmission over a Multiple-Access Channel,“ Military Communications Conference (MILCOM), 2014 IEEE, Baltimore, MD, 2014, pp. 824-829.

[9] M. Hamamura and S. Tachikawa, "Bandwidth efficiency improvement for multi-carrier systems," Personal, Indoor and Mobile Radio Communications, 2004. PIMRC 2004. 15th IEEE International Symposium on, 2004, pp. 48-52 Vol.1.

[10] I. Kanaras, A. Chorti, M. Rodrigues and I. Darwazeh, "Investigation of a Semidefinite Programming detection for a spectrally efficient FDM system," Personal, Indoor and Mobile Radio Communications, 2009 IEEE 20th International Symposium on, Tokyo, 2009, pp. 2827-2832.

[11] S. Isam and I. Darwazeh, "Design and Performance Assessment of Fixed Complexity Spectrally Efficient FDM Receivers," Vehicular Technology Conference (VTC Spring) , 2011 IEEE 73rd, Yokohama, 2011, pp. 1-5.

[12] T. Xu and I. Darwazeh, "Spectrally efficient FDM: Spectrum saving technique for 5G?," $5 G$ for Ubiquitous Connectivity (5GU), 2014 1st International Conference on, Akaslompolo, 2014, pp. 273-278.

[13] T. Xu and I. Darwazeh, "M-QAM signal detection for a non-orthogonal system using an improved fixed sphere decoder," Communication Systems, Networks \& Digital Signal Processing (CSNDSP), 2014 9th International Symposium on, Manchester, 2014, pp. 623-627.

[14] S. Isam and I. Darwazeh, "Characterizing the intercarrier interference of non-orthogonal Spectrally Efficient FDM system," Communication Systems, Networks \& Digital Signal Processing (CSNDSP), 2012 8th International Symposium on, Poznan, 2012, pp. 1-5.

[15] G. Strang, Introduction to Linear Algebra, 4th ed. Wellesley Cambridge Press, 2009.

[16] S. Takahashi, M. Hamamura and S. Tachikawa, "A demodulation complexity reduction method using $\mathrm{M}$-algorithm for high compaction multi-carrier modulation systems, "Wireless Communication Systems, 2004, 1st International Symposium on, 2004, pp. 418-422. 